\title{
TEAM-UP for quality: a cluster randomized controlled trial protocol focused on preventing pressure ulcers through repositioning frequency and precipitating factors
}

\author{
Tracey L. Yap ${ }^{1 *}$ (D), Susan M. Kennerly ${ }^{2}$, Susan D. Horn ${ }^{3}$, Nancy Bergstrom ${ }^{4}$, Santanu Datta ${ }^{5}$
} and Cathleen Colon-Emeric ${ }^{6}$

\begin{abstract}
Background: Pressure ulcers/injuries (PrUs), a critical concern for nursing homes (NH), are responsible for chronic wounds, amputations, septic infections, and premature deaths. PrUs occur most commonly in older adults and $\mathrm{NH}$ residence is a risk factor for their development, with at least one of every nine U.S. NH residents experiencing a PrU and many NHs having high incidence and prevalence rates, in some instances well over 20\%. PrU direct treatment costs are greater than prevention costs, making prevention-focused protocols critical. Current PrU prevention protocols recommend repositioning residents at moderate, high, and severe risk every $2 \mathrm{~h}$. The advent of viscoelastic (VE) high-density foam support-surfaces over the past decade may now make it possible to extend the repositioning interval to every 3 or $4 \mathrm{~h}$ without increasing PrU development. The TEAM-UP (Turn Everyone And Move for Ulcer Prevention) study aims to determine: 1) whether repositioning interval can be extended for $\mathrm{NH}$ residents without compromising PrU incidence and 2) how changes in medical severity interact with changes in risk level and repositioning schedule to predict PrU development.
\end{abstract}

Methods: In this proposed cluster randomized study, $9 \mathrm{NHs}$ will be randomly assigned to one of three repositioning intervals $(2,3$, or $4 \mathrm{~h})$ for a 4-week period. Each enrolled site will use a single $\mathrm{NH}$-wide repositioning interval as the standard of care for residents at low, moderate, and high risk of PrU development $(N=951)$ meeting the following criteria: minimum 3-day stay, without PrUs, no adhesive allergy, and using VE support surfaces (mattresses). An FDA-cleared patient monitoring system that records position/movement of these residents via individual wireless sensors will be used to visually cue staff when residents need repositioning and document compliance with repositioning protocols.

Discussion: This study will advance knowledge about repositioning frequency and clinically assessed PrU risk level in relation to PrU incidence and medical severity. Outcomes of this research will contribute to future guidelines for more precise preventive nursing practices and refinement of PrU prevention guidelines.

Trial registration: Clinical Trial Registration: NCT02996331.

Keywords: Pressure ulcer, Pressure injury, Prevention, Nursing, Repositioning

* Correspondence: tracey.yap@duke.edu

${ }^{1}$ School of Nursing, Duke University, Durham, NC, USA

Full list of author information is available at the end of the article 


\section{Background}

Pressure ulcer/injury (PrU) prevention remains a challenge within the nursing home $(\mathrm{NH})$ environment $[1,2]$. A PrU is any skin lesion over a bony prominence resulting from prolonged exposure to pressure that causes capillary occlusion and eventual tissue necrosis. Most PrUs are avoidable, but those that do develop are associated with complications (e.g., chronic wounds, amputations, septic infections, and premature deaths) [3] and with overall deterioration in prognosis, compromising both a patient's health status and quality of life. Estimated annual treatment costs (\$9.1-11.6 billion in the US [4]) are greater than prevention costs [4-8], even without considering other costs to the individual and healthcare system (outpatient visits, work loss, and lawsuits), thus making prevention a priority [9].

Residence in a NH puts individuals at risk of developing PrUs [8], and the limited mobility prevalent among $\mathrm{NH}$ residents [10] increases the intensity and duration of pressure exposure - two factors leading to PrU development [11]. The severity of illness, including signs, symptoms, physiologic parameters, and disease factors, also affects the body's response to pressure exposure and possibly increases risk for PrU development. A universally accepted approach to $\operatorname{PrU}$ prevention is to minimize pressure exposure through frequent moving/repositioning (hereafter referred to as repositioning) [12, 13] for residents clinically assessed as at-risk (typically Braden Scale $\odot$ score $\leq 18)$.

Over the last decade, NH PrU incidence rates have declined from $11 \%$ [14] to $7.8 \%$ [15]. This decline can be attributed to the advent of viscoelastic (VE) high-density foam support surfaces that reduce pressure intensity by redistributing point pressure. VE surfaces make longer intervals between repositioning possible for at-risk residents confined to bed or chair [16]. However, in order to further improve $\operatorname{PrU}$ prevention approaches [17], we must determine 1) how frequently repositioning needs to occur in order to prevent PrU development, and 2) how residents' medical severity (severity of illness), risk level (clinically assessed risk determined by the Braden Scale( $)$, and repositioning frequency affect $\mathrm{PrU}$ development individually or in combination. It is not known whether a 2, 3, or $4 \mathrm{~h}$ time interval for repositioning of residents is most effective in preventing PrUs.

Current PrU prevention repositioning protocols (derived from two small 1962 studies [18]) recommend repositioning residents at moderate, high, and severe-risk every $2 \mathrm{~h}$. With VE surfaces, it may be possible to reduce repositioning frequency by extending the repositioning interval (time elapsed between scheduled repositioning of residents) from 2 to 3 or even $4 \mathrm{~h}$ without an increase in PrU development. In the last decade, only two studies have examined repositioning protocols. One study reported fewer PrUs in residents using VE surfaces with a 4-h repositioning interval than in residents who used non-VE surfaces with more frequent repositioning $[19,20]$. The second study, Turning for Ulcer ReductioN (TURN) trial, found no significant differences in PrU incidence between 2, 3, or 4-h repositioning intervals in a sample of moderate and high-risk residents using VE surfaces. In these two studies, $90 \%$ of all new PrUs occurred within the first 15 days [20]. However, new PrUs cannot be attributed to any single factor [21], and these analyses did not comprehensively examine changes in residents' PrU risk levels or their physiological and disease factors (medical severity).

We will examine the effects of repositioning frequency (using assigned repositioning intervals of 2,3 , or $4 \mathrm{~h}$ ) on residents at low, moderate, and high risk of developing PrUs (Braden Scale score 10-23) and will explore the effects of risk level and medical severity individually and in combination over a 4-week intervention period. Residents deemed to be at low risk are not commonly studied, but they too develop PrUs [2, 14-16] and are therefore included in this study. An FDA-cleared patient monitoring (PM) system using individual wireless triaxial accelerometer sensors will record resident position and movement, automate repositioning schedules, provide visual cues to nursing staff [22] via electronic display screens at the nursing station and unit hallway(s) that indicate when each resident needs repositioning, and confirms completion of repositioning. This system provides a timestamp that repositioning occurred and addresses concerns about nursing electronic medical record (EMR) documentation compliance.

The specific aims of the TEAM-UP (Turn Everyone And Move for Ulcer Prevention) study are to:

Aim 1: Determine differences in the incidence of new pressure ulcers $(\mathrm{PrU})$ in nursing home $(\mathrm{NH})$ residents at low, moderate, and high risk using VE support surfaces and repositioned at intervals of 2,3 , or $4 \mathrm{~h}$, in nine randomly assigned NHs over a 4-week period, using the patient monitoring (PM) system to determine movement.

Aim 2: Determine how medical severity components (as measured by a modified Comprehensive Severity Index [23, 24]), changes in clinically assessed risk level (low, moderate, high as measured by the Braden Scale $\odot$ [25]), assigned repositioning intervals, and their interactions are associated with development of PrUs, controlling for resident demographics.

Exploratory Aim: Evaluate PrU prevention intervention approaches in $\mathrm{NH}$ groups repositioned at 2, 3, or 4-h by: 1) comparing the intervention resource costs (VE support surfaces, PM system service/sensor use, fixed and variable labor costs for training and repositioning) 
and incremental cost-effectiveness ratio of cost per \% reduction in PrUs, and 2) exploring staff and resident satisfaction with intervention approach.

\section{Significance}

PrUs occur most commonly in older adults [6], and $\mathrm{NH}$ residence is a risk factor [8] for development. At least one of every nine U.S. NH residents will experience a PrU $[14,26]$ and many NHs have high incidence and prevalence rates, in some instances well over $20 \%[27,28]$. The estimated cost of treating PrUs ranges from $\$ 21,000-152,000$ per PrU [29]. Effective prevention can reduce PrU incidence and avoid treatment costs while improving resident satisfaction, safety, and quality of life. Residents identified as moderate- or high-risk (who comprised $22-28 \%$ and $56-57 \%$ respectively of the $\mathrm{NH}$ population in two recent studies [12, 30]) are considered most likely to develop PrUs; however, low-risk NH residents also develop PrUs [19, 28, 31] and must be considered in any comprehensive prevention approach. PrUs are challenging to address, given the variations in $\mathrm{NH}$ size, staffing, and resident diversity, but creating cost-effective solutions is imperative because 1 in every 5 adult Americans (some 72 million) will be $>65$ years old by 2030 and PrUs could increase exponentially [6].

PrUs remain a multifactorial problem, although the advent of innovative products (e.g., VE support surfaces) has minimized some risk. In order to further reduce PrU incidence, it is important to both 1) test and challenge current prevention protocols to identify a gold standard, and 2) understand the intersection of multiple contributing factors to PrUs to identify additional opportunities for improvement. Improving prevention protocols is critical on multiple levels (i.e., individual, department, facility, society) given the cost of treating PrUs, the direct impact on both staff and residents, and the fact that overall costs of delivering prevention protocols is less than treatment costs.

The TEAM-UP study challenges the idea that repositioning must be completed every $2 \mathrm{~h}$ to best prevent the onset of PrUs. It builds on prior evidence from an intervention study $[19,20]$ showing that repositioning intervals may be extended from every $2 \mathrm{~h}$ to every 3 or $4 \mathrm{~h}$ without increasing PrU incidence, and attempts to expand the evidence base for PrU prevention by determining how residents' medical severity, clinically assessed PrU risk level, and assigned repositioning interval affect PrU development individually or in combination. TEAM-UP extends previous study periods $[19,20]$ to $4-$ weeks ( 28 days) to help determine the optimal resident repositioning interval, and will: 1) place all $\mathrm{NH}$ residents on VE (high-density foam) support surfaces to avoid point pressure and redistribute pressure, so as to enable longer periods between repositioning; 2) enroll residents at low risk for PrU development in addition to those identified as moderate or high risk; 3) use a PM system with individual wireless sensors to track repositioning compliance with time-stamped documentation and provide repositioning reminders, while also providing automatic feedback for individual nursing staff and the team to improve communication; and 4) monitor fluctuations in resident characteristics and risk levels, which may be associated with development of PrUs. The study intervention will be carried out by $\mathrm{NH}$ staff in real-life practice conditions with protocol and training support, rather than by an external intervention team. The impact of the intervention on the occupational subculture of the nursing staff will be explored, as will the perspectives of both nursing staff and residents. Nurse champions will be trained to support staff as resources for protocol implementation and the use of the PM system and sensors.

\section{Methods}

The TEAM-UP study is a cluster randomized clinical trial with facility-level use of an assigned repositioning interval as the primary intervention and will be conducted at $9 \mathrm{NHs}$ that are all using VE support surfaces. Each participating $\mathrm{NH}$ will be randomly assigned to one of three intervention arms. Each arm will have $3 \mathrm{NHs}$ with each $\mathrm{NH}$ using a single, $\mathrm{NH}$-wide repositioning interval at either 2,3 , or $4 \mathrm{~h}$ as standard of care during the 4-week intervention period. Randomization will occur at the facility level. The sequence in which the repositioning intervals are to be implemented will be randomly ordered and, then assigned to $\mathrm{NHs}$ inclusive of each arm of NHs. Thus, 3 clusters of $3 \mathrm{NHs}$ are created with randomly sequenced repositioning intervals.

A secondary cluster is created within each $\mathrm{NH}$ as the $\mathrm{NH}$ implements its standard risk assessment protocol; residents will be clinically categorized forming clusters by level of risk for developing a new PrU. Level of risk categorization into 3 groups is defined by a Braden score of low (15-18), moderate (13-14), or high (10-12) [32]. All eligible residents will wear a wireless triaxial accelerometer sensor throughout the intervention as part of the NH's standard of care. Residents may refuse the sensor and can decline to move when prompted by staff as part of their right to refuse care (see Fig. 1). This pragmatic approach offers a unique opportunity to study a single $\mathrm{NH}$-wide repositioning schedule, thus avoiding the potential for staff confusion associated with resident-level random assignment to repositioning intervals, which would require staff to simultaneously execute multiple turning schedules.

Medical severity components and clinically assessed risk level will be extracted from the EMR and examined for assessment-to-assessment change, thus allowing 


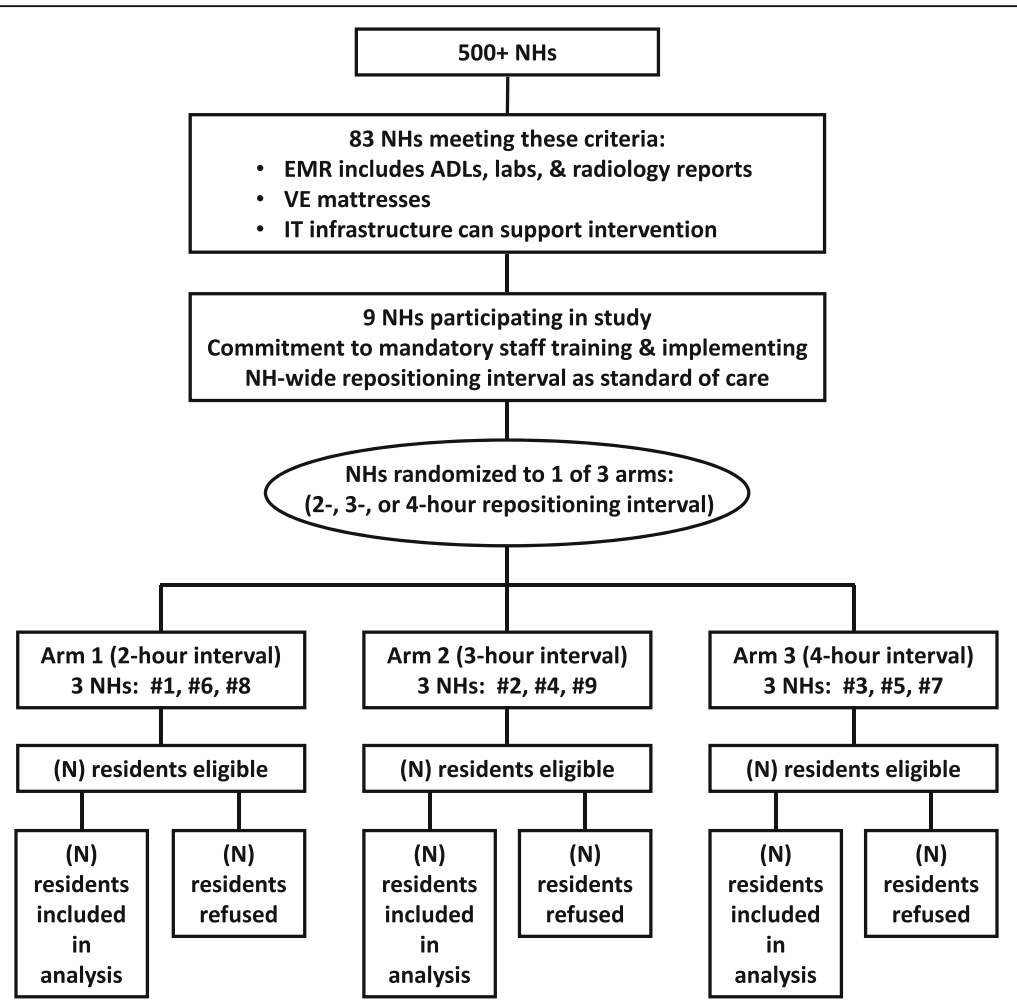

Fig. 1 Flowchart for study protocol manuscript

identification of medical severity components associated with development of a new PrU. We will use intervention resource cost elements (VE surfaces, PM system and sensor service, fixed and variable labor costs for training and repositioning) to assess implementation cost and calculate a cost-effectiveness ratio per \% PrU reduction. $\mathrm{NH}$ nursing staff will be asked to complete a nursing culture assessment web-based survey administered electronically at their $\mathrm{NH}$ pre- and postintervention. Focus groups will be conducted using semi-structured questions framed according to the Diffusion of Innovation model to gather/assess $\mathrm{NH}$ staff and resident perspectives of each group's satisfaction regarding the repositioning interval and challenges experienced in use of the PM system.

Additional information is available on the study website (https://teamup.nursing.duke.edu).

We are funded to conduct this study from 07/01/2016 to $03 / 31 / 2021$. We have completed implementation in 3 of the 9 nursing homes and additional study data collection and analyses will be completed by 03/31/2021.

\section{Sample, setting, recruitment}

\section{NH sample}

Researchers established a collaboration with a United States, for-profit, Medicare-certified, intermediate and skilled NH system with $>450 \mathrm{NHs}$ in over 30 states. A convenience sample of $9 \mathrm{NHs}$ will be selected from volunteer facilities within the system if they have $>100$ beds and are using viable VE mattresses examined 2 months prior to intervention implementation, and have full EMR use that includes activity of daily living (ADL) documentation and laboratory and radiology electronic results. Participating NHs must agree to implement the patient monitoring system technology with a $\mathrm{NH}$-wide repositioning protocol, and agree to a mandatory nursing staff in-service training provided by the research team. Full-time and part-time nursing staff (RNs, LPNs, CNAs) working clinically with residents will participate in an $\mathrm{NH}$-mandated education session to explain the study protocol and prepare them regarding PM system use. NH's will be assigned in random order to a repositioning interval.

The NH will give all its residents/family an information sheet that explains the study and $\mathrm{NH}$-wide adopted repositioning protocol and informs them of their right to refuse care and/or request a more frequent repositioning interval. The information sheet will also explain risks, benefits, and what is involved in participation.

\section{Resident sample}

All residents ( $\geq 18$ years old) using a VE mattress (support surface) who are without PrUs and adhesive allergy and are clinically assessed as low, moderate, or high risk 
for new PrU development will participate in their respective $\mathrm{NH}$-wide repositioning frequency protocol and receive standard $\operatorname{Pr} U$ prevention care.

$\mathrm{NH}$ resident participants will include those residents at the time of study initiation and any residents newly admitted during the 4-week study period. All individuals who are $\mathrm{NH}$ residents for a minimum of 3 days and meet inclusion criteria will be included in data analyses, because superficial PrUs have been reported to occur within several hours to days following $\mathrm{NH}$ admission, and "deep tissue injury", a type of PrU resulting from pressure on muscle and fascia is thought to appear on the surface of the skin approximately 3 days following exposure to pressure.

A waiver of informed consent has been obtained from the Primary Investigator's (PI) Institutional Review Board. Data on newly admitted residents with less than a 3-day stay will not be included in the analysis, because deep tissue injuries that result from pressure prior to admission may not be visible for up to 3 days after pressure exposure. Residents will also be excluded from the analysis if PrUs are present at baseline, PrU risk is severe (Braden score $\leq 9$ ), or if the resident is cared for on a non-VE specialty surface, has "do not turn orders" present, or has an allergy to the adhesive used on the sensor. Residents assessed as being at severe risk of developing a $\operatorname{PrU}$ (estimated $\leq 10 \%$ per $\mathrm{NH}$ ) will be excluded because they typically require specialty surfaces and individualized and frequent repositioning, and their health status is generally more unstable. Data on all other residents will be included, using an intention-totreat approach. The target $\mathrm{N}$ for analysis is 951 ; we aim to enroll 1100 residents at baseline. Residents who are discharged, transferred, or die will be included in the intention-to-treat analysis.

\section{Staff sample for survey}

$(N=1200)$ All nursing staff (registered nurses, licensed practical nurses, and certified nursing assistants) working clinically full- or part-time with residents will be eligible irrespective of job category at each $\mathrm{NH}$. Will be asked to complete an anonymous web-based electronic survey via touch screen tablet.

\section{Focus group(s) sample}

Two focus group sessions (one of residents and one of staff; six participants/ group), will be conducted at three selected NHs (one NH per arm) to evaluate implementation and perspectives regarding approach. Using convenience sampling, English-speaking intervention participants will be eligible to join, irrespective of gender, race, ethnicity, and physical ability.
1. Residents. Residents (aged $>18$ ) who are cognitively aware and willing to participate will be selected and consented to participate in one focus group per selected NH (total of three groups; $n=18$ residents). Participants may have refused sensor monitoring.

2. Staff. Nursing staff (aged $>18$ ) working clinically full- or part-time with residents will be selected irrespective of job category and consented for one focus group per selected $\mathrm{NH}$ ( 3 groups; $n=18$ nursing staff).

\section{Risk and challenges}

This study design includes NHs with the same corporate management to ensure that the same standard-of-care protocols are in place in each $\mathrm{NH}$. A major challenge for $\mathrm{NH}$ research is the potential for staff turnover. Using a successful strategy from our prior studies [31], we secured a written commitment that the study will continue even if there is a change in one or more of corporate administrative leaders for the $\mathrm{NH}$ system. We also have designed the study to be robust to staff turnover and potential staff resistance to a new repositioning schedule, by incorporating mandatory training with supplemental on-site instruction as needed and also incorporating training into the NH's new staff orientations. Another potential challenge is staff inconsistency in implementing the repositioning protocol standard-of-care; consistency in repositioning is important to being able to subsequently demonstrate that the repositioning at the assigned interval actually occurred [33]. In fact, in practice, extended repositioning intervals already are thought to be extended beyond $2 \mathrm{~h}[34,35]$ and there are longstanding concerns that nursing staff documentation may be unreliable for use as evidence of repositioning [20, 31, 36, 37]. The PM system monitoring capabilities will enable concerns about repositioning protocol compliance and documentation to be addressed via time-stamped tracking of identified position changes.

\section{The intervention}

Elements of the intervention include a $\mathrm{NH}$-wide assigned repositioning interval 2,3 , or 4-h) with repositioning of residents using viable VE mattresses. Monitoring and documentation of repositioning intervention will be accomplished with use of a PM system. Table 1 describes the protocol components in detail.

\section{Repositioning protocol}

Residents who are in bed will be repositioned according to the predetermined $\mathrm{NH}$-wide assigned repositioning interval of every 2,3 , or $4 \mathrm{~h}$, and rotated from side to back to side. In accordance with International PrU prevention guidelines [13], residents will be positioned in the lateral position at no more than a $30^{\circ}$ tilt. While in 
Table 1 Protocol for implementation of intervention approach

\begin{tabular}{llll}
\hline Protocol Components & Rationale/Outcome & Persons Responsible & Time (Fig. 4) \\
\hline (1) PrU Prevention and Study Protocol Education & Establish knowledge of PrU etiology & RNs, LPNs, NAs, NPs & Initial session week prior to \\
Content: Introduces all NH nursing staff to PrU & prevention practices, \& study protocol. & repositioning protocol start; \\
etiology, evidence-based PrU prevention & Facilitate consistency in response to & for new staff orientation: one \\
practices, benefits of repositioning, \& EMR & intervention protocols, safety, \& group & 60-min session followed by \\
documentation. Outlines roles \& & effectiveness. & one-on-one training as \\
responsibilities, staff work flow, \& performance & Encourage problem-centered thinking & needed when working. \\
\& documentation of movement of residents. & \& learning by demonstration and &
\end{tabular}

$\&$ documentation of movement of residents.

Content includes $\mathrm{NH}$-wide repositioning

strategy \& suggestions for best practice

implementation with cueing every 2,3 , or $4 \mathrm{~h}$

(24 h/day, 7 days/wk) depending on NH's

hourly protocol.

Delivery Strategies:

- Researcher leads initial session containing

videos embedded in PowerPoint overview

with simulation of repositioning \&

documentation process, followed by

questions \& answers.

- Reference cards provided to all staff summarizing PrU etiology \& staff responsibilities in relation to study protocol, day-to-day procedures, \& directions for carrying out the protocol \& completing study measures \& documentation.

(2) Champion Training

Champion Training Class:

Champions will learn to facilitate the

educational component \& practice mentoring on study strategy, problemsolving about protocol implementation, EMR documentation, \& observation checklist completion; Leaf sensor placement with system activation check \& skin monitoring. Support by Research Facilitators:

A researcher will contact each champion every week via phone or videoconferencing during the intervention for support, advising, or refreshers as needed: champions will also have a phone number to call to seek help from research staff as needed.

(3) Patient Monitoring System Training Focus is on patient monitoring, responding to the 2-, 3-, or 4-h visual cues on the screen displays located at nursing station and in hallway on clinical unit, and review sample data feedback \& practice interpretation.

Placement of Sensor on Resident:

Focus is on licensed nurse \& process for sensor placement and oversight of CNA routine observation. Staff will learn to place sensor and ensure its activation and to remove and discharge sensor. Leaf placement will be periodically checked by champions to ensure fidelity.

(4) VE Support Surface Audit

This will be completed at each $\mathrm{NH}$ site according to NH system's policy. Each NH will provide audit documentation demonstrating that all mattresses are adequate and have been examined (those that were not adequate were replaced) within the last year according to NH system's Mattress and Safety Audit Guidelines.
Prepare champions to mentor staff, build trust, maintain consistency, and facilitate sustained implementation.

Staff will be prepared to monitor the screen display for repositioning status (cue \& next repositioning times) and to complete the appropriate documentation.

Ensure viable mattresses in use prior to intervention start
Volunteer nursing staff Week prior to repositioning protocol start: one 60-min session.

RNs, LPNs, NAs, NPs

Week prior to repositioning protocol start:

Part of Pru Prevention Protocol \& Education session followed by one-on-one on-the-job training as needed.

Research team and

Minimum of 1 month prior to repositioning protocol start. 
the supine position, the head of the bed may be elevated during feeding, but the duration of elevation greater than $30^{\circ}$ will be limited to avoid exposure to friction and shear. Residents will wear pressure reduction boots if ordered by the $\mathrm{NH}$; however, because there is no evidence that these perform better than elevating the heels using a pillow, the latter will be standard procedure in this study. According to $\mathrm{NH}$ policy, there is a standing order that nursing staff should assist non-bedfast residents while in the chair to stand/move/reposition each hour to relieve ischial pressure, preventive seating cushions will be used as necessary according to NH guidelines. PrUs resulting from sitting will appear on ischial tuberosities; these PrUs are clearly different from PrUs on the sacrum/coccyx, trochanter, and heels that occur when lying in bed. Repositioning devices (e.g., turning sheets or trapeze) will be used as appropriate to reduce friction and shear, and pillows and foam wedges will be used to maintain position according to international guidelines.

In $\mathrm{NHs}$, up to $9 \%$ of long-stay residents spend the majority of most days in bed or in a chair in their room [38]. Bates-Jensen et al. found that most residents spent $17 \mathrm{~h}$ a day in bed [34]. We expect, however, that $90 \%$ of residents will be sitting for at least part of the day. Repositioning will occur during the time that residents are in bed. Figure 2 is a visual depicting the required assessment and repositioning safety and skin care check to be performed by nursing staff and the criteria that must be met at each decision point in implementing the repositioning protocol.

\section{VE support surface}

All NH's VE support surfaces (mattresses) in this study will meet or exceed international guidelines [39] for the minimum acceptable requirements for density and support to ensure that a resident's mattress is flexible enough to deflect pressure over bony areas that come in contact with the mattress. VE surfaces will be examined 2 months prior to each NH's intervention and all mattresses that are not viable will be replaced prior to study implementation.

\section{The patient monitoring (PM) system}

A wireless system for monitoring resident position and movement will be used to visually cue nursing staff on electronic display screens to ensure protocol adherence and to evaluate $\mathrm{NH}$-repositioning protocol delivery fidelity [40]. This system is used in hospitals and NHs to enable caregivers to implement individualized repositioning protocols and it drives compliance with the care plan by visually notifying staff when repositioning is required $[7,41,42]$. Using a skin-sensitive medical grade adhesive backing (similar to an EKG electrode), a wireless, wearable single-patient use sensor will be placed by nursing staff on the resident's upper chest beneath the clavicle on either the right or left side at approximately the midclavicular line (at least $4 \mathrm{in}$. away from a pacemaker or implantable cardioverter-defibrillator). The individual sensor has no on- or off-switch and activates automatically when the opaque backing is removed to expose the sensor to light. Each sensor will be associated with a specific resident, will have individualized repositioning parameters such as the 2-, 3-, or 4-h interval, and will track and indicate changes in body position. The PM system's turn management software displays each resident's repositioning history and current positional status in an organized and simple way on strategically placed display screens at the nursing station and mid-point in each hallway, making staff aware when repositioning actions are becoming necessary or are overdue.

\section{Treatment fidelity}

Our treatment fidelity protocols use the National Institutes of Health Behavior Change Consortium's [43] model. To ensure intervention integrity, repositioning of residents will be assigned to and performed by nursing staff. Each resident's repositioning activity will be monitored with a wireless sensor and documented within the PM system, and randomly scheduled weekly observation will be conducted of staff turning/repositioning selected residents (6 per week $\mathrm{x}$ a minimum of 4 weeks) to provide a fidelity check of the system's accurate detection of turning/repositioning activities. Studies and clinical data show that the device effectively detects position changes according to threshold parameters, like degree or angle of position change $[44,45]$. Each $\mathrm{NH}$ will use a single $\mathrm{NH}$-wide repositioning interval as the standard of care for low-, moderate-, and high-risk residents. This pragmatic approach offers a unique opportunity to study a single NH's facility-wide repositioning schedule, thus avoiding the potential for staff confusion if more than one schedule is executed within a facility. Furthermore, each repositioning interval will be tested in three $\mathrm{NHs}$ using VE surfaces, enabling replication of findings.

\section{Data collection \& storage (see Fig. 3 and Table 2) Quantitative data collection procedures}

NH-level data. NH Company has agreed to provide each participating NH's characteristics, including bed size and nursing staff hours, facility-level data (PrU period prevalence and incidence, staff number, mix, and turnover) at baseline (12 months prior) and during the intervention period. Staff mix, wage, and fringe benefit rates will be also be obtained. Data will be collected from publicly available sources (https://www.medicare.gov/nursinghomecompare/ search.html). These data will be used as covariates in the multivariate analyses. 


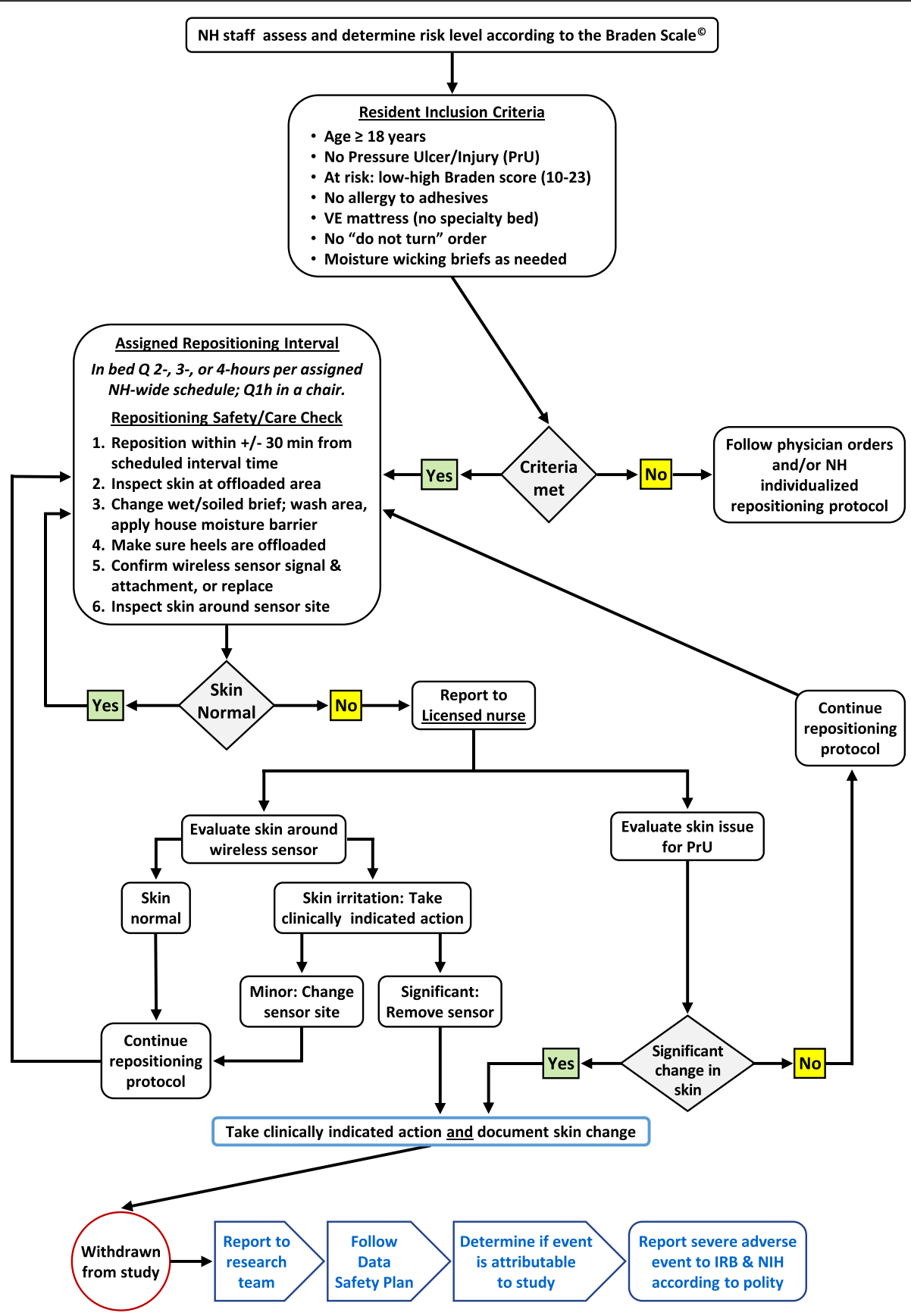

Fig. 2 Repositioning and skin safety decision tree

Resident-level data. Resident data extracted from the PM system database and the 12-month baseline and 4week intervention MDS (batch file) and EMR; each resident will be assigned a study ID number prior to data extraction. New PrU incidence will be collected during the intervention period. Baseline data will be collected for 12 months just prior to intervention launch and will consist of EMR, MDS, and PrU incidence (Table 2) data. Intervention data extraction (of EMR, MDS, and PM system data) will occur weekly during the intervention 4-week period. The PM system will schedule resident repositioning frequency and gather resident repositioning sensor data automatically according to $\mathrm{NH}$ standards of care. Data collected by each resident's sensor will be automatically communicated wirelessly through a proprietary mesh network of relay antennas to the respective NH's Central Monitoring Station containing turn management software. Wireless sensor data on all monitored residents will be stored by the PM system. The wireless sensor number will become associated with a resident 


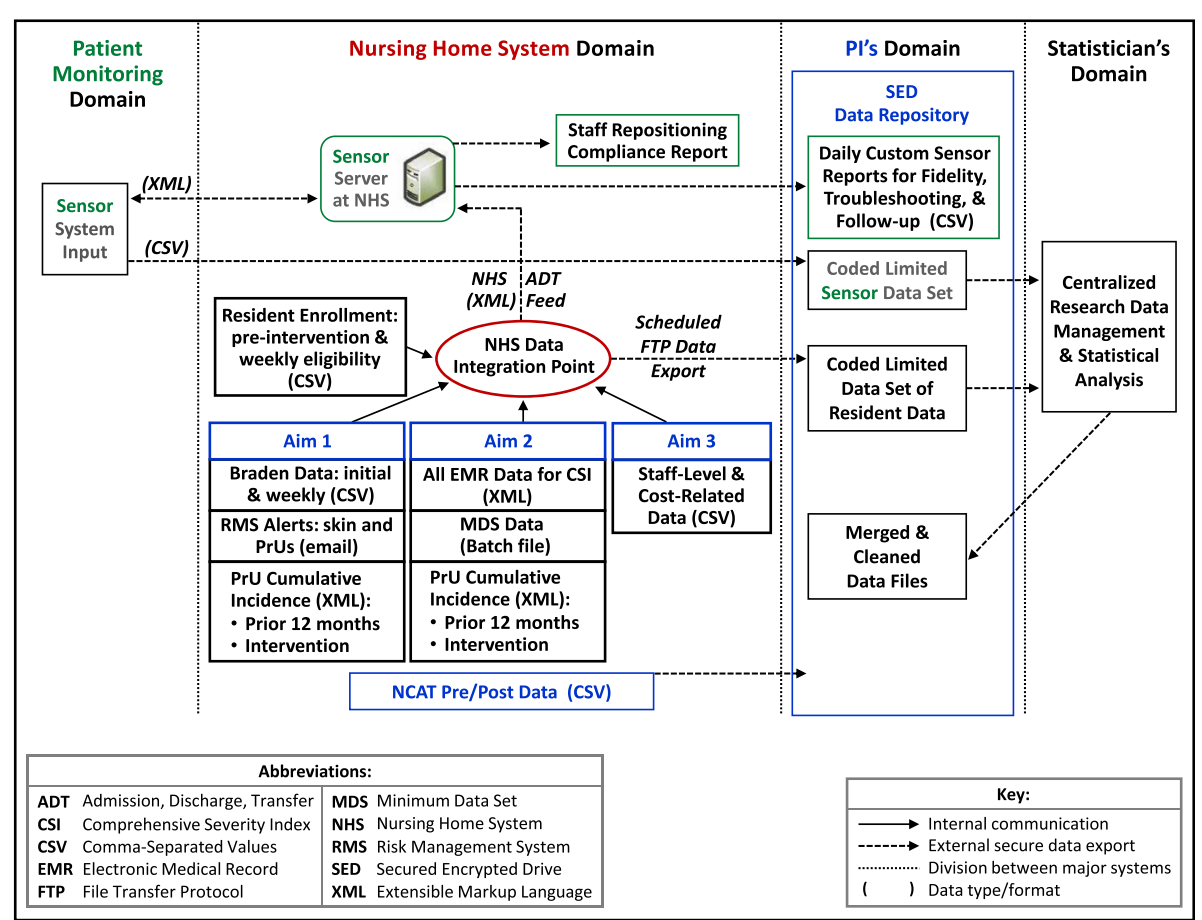

Fig. 3 TEAM UP study high-level components diagram and key data sources

study identifier to be created as described below. The study ID number will be used to facilitate data comparisons. Wireless sensor data for all monitored residents will be securely stored in the PM system database.

Bench testing and clinical trials of the PM system's wireless sensor have demonstrated it to be a valid measure of active and passive movement (repositioning). The sensor's sensitivity accuracy is $+/-2.5 \%$ consistent with the industry standard for a linear triaxial accelerometer. Studies and clinical data show that the device effectively detects position changes according to threshold parameters, like degree or angle of position change. Wireless sensor data on all monitored residents will be securely stored in the patient monitoring system database. Wireless sensor data along with other resident data, including EMR data, will be transferred to the researchers for analysis. Turn management software in the PM system displays each resident's repositioning history and current positional status in an organized and simple way on strategically placed nursing station and hallway display screens, making staff aware when resident repositioning actions are becoming necessary or are overdue. The percentage of on-time repositioning will be calculated for the period during which the wireless sensor is worn.

\section{EMR integration of patient monitoring system and CSI} procedures. The EMR will be used to facilitate data collection and export of study-related data, which includes an interface with wireless sensor data. This approach eliminates costly labor-intensive, in-person fidelity checks and minimizes research burden on staff. Individual wireless sensors will communicate with wireless antennas placed throughout the $\mathrm{NH}$, permitting resident monitoring in all $\mathrm{NH}$ areas. The patient monitoring system will allow us to ascertain repositioning protocol adherence by measuring consistency between scheduled, performed, and documented repositioning frequency with random weekly observational repositioning checks to ensure repositioning fidelity. With CSI and other resident data, medical severity components will be assessed by staff based on $\mathrm{NH}$ policies and procedures and monitored in relation to risk level.

Staff-level data. The web-based survey will be administered electronically on-site with touch screen tablet, pre/ post the 4-week intervention during the time the employee is at work. The Nursing Culture Assessment Tool (NCAT) $[46,47]$ survey contains 19 -items and there are some demographic items (i.e., length of time employed, job category, age, gender, education level, shift), and will be administered pre/post intervention. At the time of survey data collection, data will be transferred through a secured server from which it will be downloaded into a database for statistical analysis. Data will be stored on the study's secure encrypted drive (SED).

\section{Measures}

The measures and time points at which data will be collected are summarized in Table 2, and Fig. 4 is the study timeline and data collection schedule. 
Table 2 Summary of concepts and instruments / measurements used to collect data

\begin{tabular}{|c|c|c|}
\hline Concept Measured & Measures / Instrumentation & Source (Fig. 3) \\
\hline $\begin{array}{l}\text { Cumulative incidence of (new) PrUs for } \\
\text { pre-intervention (baseline) period }\end{array}$ & $\begin{array}{l}\text { Cumulative Incidence rate }=[(\# \text { of residents with } 1 \text { or } \\
\text { more new PrUs during a } 1 \text {-month period }) \div(\text { of } \mathrm{NH} \\
\text { residents with } 3 \text { or more day stay) }] \times 100 ; \text { calculated }\end{array}$ & $\begin{array}{l}\text { - MDS } 3.0 \\
\text { - NH self-report } \\
\text { - EMR data extract }\end{array}$ \\
\hline
\end{tabular}

Cumulative incidence of (new) PrUs for intervention period

Braden Scale@

Repositioning frequency

Recorded repositioning frequency

Repositioning protocol adherence: $\%$ agreement

Medical Severity —Comprehensive Severity Index (CSI)

Clinically assessed risk level (Braden Scale) monthly to establish baseline over the 12-month period before each $\mathrm{NH}$ intervention begins.

Cumulative Incidence rate $=[(\#$ of residents with 1 or more new PrUs during intervention period) $\div$ (\# of residents participating in intervention for 3 or more days) $\times$ 100; calculated for the full 4-week intervention period.

The Braden scale is being used per protocol by participating $\mathrm{NH}$ s to assess resident risk for PrUs at intervention start and weekly thereafter.

The scheduled duration of time for a resident's pressure exposure as reflected by the 2-, 3-, or 4-h time interval at which the resident is repositioned; each $\mathrm{NH}$ will be randomly assigned to one of the study's 3 arms and will use a single $\mathrm{NH}$-wide repositioning frequency.

The actual time interval at which repositioning occurs, as documented by the Leaf sensor; recorded in clock time hours and minutes, $24 \mathrm{~h}$ per day, from start to end of resident's participation in the 4-week intervention. Repositioning activities of residents will be captured using the Patient Monitoring System; Admission, Discharge, Transfer (ADT) feed from NHS to Monitoring System enables a match for Study ID\# to resident movement, which is required for resident tracking in the event of room change, discharge/ readmission, or other events.

Percentage of agreement between scheduled repositioning frequency and repositioning frequency recorded by wireless sensor:

[(scheduled clock time - recorded clock time $=\#$ minutes difference) $\div 60] \times 100]$; calculated for each resident's repositioning episode.

Calculated score on severity resulting from distillation of physiologic parameters, signs, symptoms, laboratory results, physical findings, and diagnoses, using the modified Comprehensive Severity Index (CSI), a risk adjustment system. The more abnormal the signs and symptoms, the higher the severity score: Level 1 is normal to mild, and Level 4 is catastrophic, life-threatening, or likely to result in organ failure.

Individual components thought to influence a resident's skin tolerance to pressure exposure will be monitored from assessment to assessment for Leaf, resident care parameters, CSI, and Braden score, with data extracted from EMR and the most recent MDS 3.0 assessment (initial, annual, quarterly, or change in status).

Total summed score (range 6 to 23) on the Braden Scale for Predicting Pressure Sore Risk $\odot$, comprised of 6 subscales (sensory perception, mobility, activity, moisture, nutrition, and friction \& shear). Braden Score will be categorized as low (15-23), moderate (13-14), or high (10-12) risk. Collected weekly during intervention. Routinely collected by $\mathrm{NH}$ on admission, weekly $\times 4$, quarterly, and upon suspected change in health condition. Change in category will be extracted.
- MDS 3.0

- $\mathrm{NH}$ self-report

- EMR data extract

- EMR data extract

- PMS Central Server containing Monitoring Management Software

- PMS Central Server containing Monitoring Management Software
- PMS Central Server containing Monitoring Management Software

- MDS 3.0

- EMR data extract
- EMR data extract 
Table 2 Summary of concepts and instruments / measurements used to collect data (Continued)

\begin{tabular}{|c|c|c|}
\hline Concept Measured & Measures / Instrumentation & Source (Fig. 3) \\
\hline Culture Assessment & $\begin{array}{l}\text { Demographic \& NCAT data will be used to assess the } \\
\text { basic characteristics of participants and healthcare } \\
\text { setting and clinical area worked. Data to be collected } \\
\text { from each participant include, Length of time } \\
\text { employed, job category, age, and gender. }\end{array}$ & - Qualtrics survey on iPads \\
\hline Staff mix & $\begin{array}{l}\text { The number of RNs, LPNs, and CNAs who work at } \\
\text { each NH per day each day during the 4-week } \\
\text { intervention at that } \mathrm{NH} \text {, and for } 12 \text { months prior to } \\
\text { intervention start. }\end{array}$ & • NH self-report \\
\hline Staff turnover rate & $\begin{array}{l}{[(\text { Number of nursing staff }(C N A, L P N, \& \text { RN) who leave }} \\
\text { during the time period }) \div \text { (Number of nursing staff at } \\
\text { beginning }+ \text { Number of nursing staff at the end } \div 2)] \\
\times 100 \text {. }\end{array}$ & - NH self-report \\
\hline $\begin{array}{l}\text { Labor cost: } \\
\text { 1) Training } \\
\text { 2) Conducting repositions }\end{array}$ & $\begin{array}{l}\text { 1) Time needed for training nursing staff multiplied } \\
\text { by respective wage \& fringe rates of training } \\
\text { participants, 2) number of repositioning's for each } \mathrm{NH} \\
\text { and study arm and time to conduct repositioning } \\
\text { collected by wireless sensor system; multiplied by } \\
\text { nurse wage \& fringe rate. }\end{array}$ & - NH self-report \\
\hline Non-labor cost inputs & $\begin{array}{l}\text { Market prices paid fully depreciated over their } \\
\text { respective useful life will be used to calculate daily } \\
\text { equipment cost rates. Includes VE surfaces, Leaf } \\
\text { services, and sensor use. }\end{array}$ & - NH self-report \\
\hline
\end{tabular}

\section{Qualitative data collection procedures}

Focus groups. In three randomly selected $\mathrm{NHs}$, one from each hourly repositioning protocol, focus group sessions (one for staff, one for residents) will be held to explore the intervention approach and implementation. There will be one group of NH staff $(n=6)$ and one group of $\mathrm{NH}$ residents $(n=6)$ per site. NH staff focus group discussion will be stimulated using semistructured questions to gather participant perspectives on implementation challenges. Each session will last 30$45 \mathrm{~min}$. At the start of all focus group sessions participants will be consented. All focus groups will be recorded and verbatim transcripts (with no participant identifiers) will be prepared for subsequent use in data analysis.

\section{Analysis}

Quantitative analyses. We hypothesize that there will be no significant difference in $\operatorname{PrU}$ incidence between the 3 treatment arms in the study using three risk level groups: high risk (Braden Scale Score, 10-12), moderate risk (Braden Scale Score, 13-14), and low risk (Braden Scores $>14$ ). The hypothesis of no group difference will be tested by examining whether the $95 \%$ confidence intervals of the rates of $\mathrm{PrU}$ and the 2-h repositioning overlap. If so, the hypothesis for no group difference will be confirmed. We will also determine how changes in medical severity components (as measured by a modified Comprehensive Severity Index), change in clinically assessed risk level (low, moderate, high as measured by

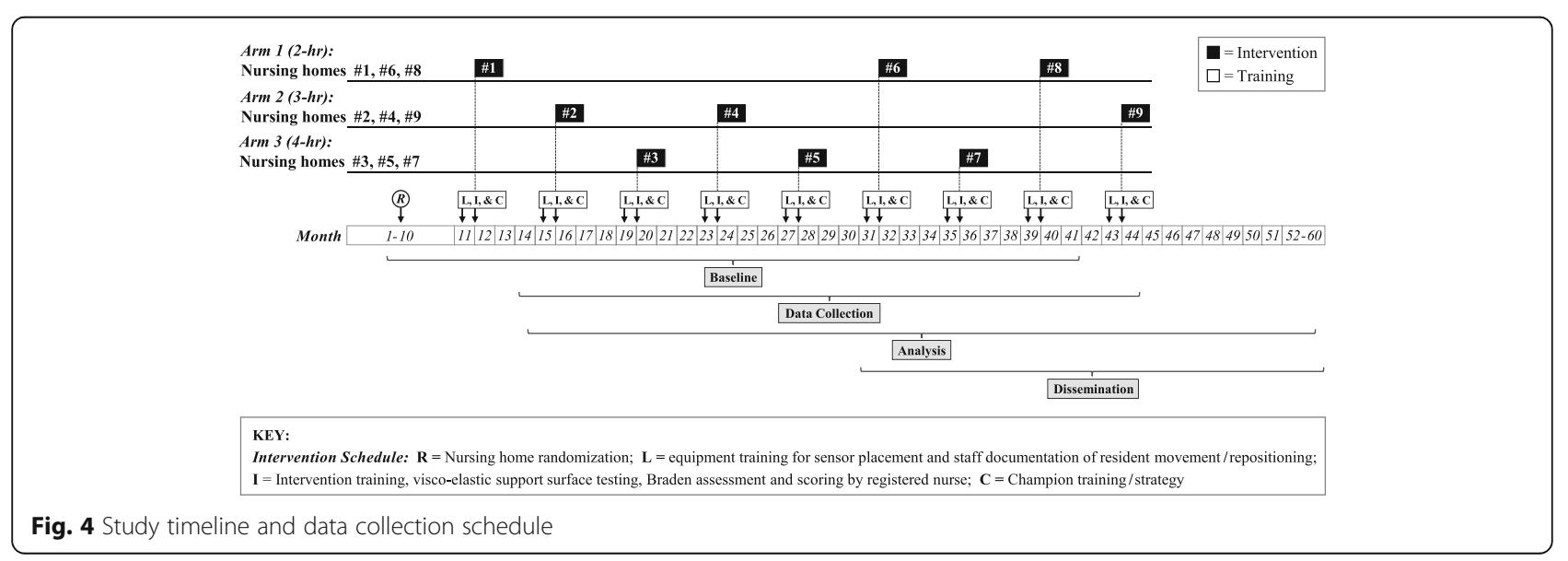


the Braden Scale) and their interactions with repositioning schedule are associated with $\operatorname{PrU}$ development.

Descriptive statistics will be used to examine resident status, interventions, outcomes, and cost elements. For categorical variables, we will create contingency tables and use chi-squared or Fisher's exact tests to determine the significance of bivariate differences. For continuous variables, we will use analysis of variance or nonparametric tests, depending on variable distributions. A two-sided $p$-value $<0.05$ will be statistically significant. Intervention cost by $\mathrm{NH}$ and study arm and incremental cost-effectiveness ratios (ICERs) will be calculated and compared for cost per percentage reduction in PrUs. Sensitivity analysis will be conducted with the range of observed repositioning times, nurse salaries, and market prices for non-labor inputs to assess the robustness of the ICERs and their sensitivity to labor and non-labor input prices.

Because we will be examining PrU development from assessment to assessment, the rate of $\mathrm{PrU}$ incidence will be modeled as a binary outcome using repeated measures logistic regression and a specified working correlation matrix that models the correlation of responses within subjects. Full likelihood estimation methods are not appropriate for repeated measures logistic regression, so generalized estimating equations (GEEs) will be used instead with model fit assessed using the quasilikelihood information criterion (QIC). Robust standard errors, clustered at the facility level, will be employed to account for covariance among residents within facilities over time. To test the robustness of models, we will also use non-linear mixed models using PROC GLIMMIX as another option for modeling the data (instead of GEEs using PROC GENMOD). With PROC GLIMMIX, we can use random effects to model the covariance rather than clustered standard errors. As predictors, we will include time-invariant resident characteristics, e.g., age, gender, Medicaid payer, and repositioning schedule, as well as time-varying characteristics, such as resident severity measure, risk level as determined by Braden Scale` score, staffing characteristics (e.g., number, mix, and turnover), and suggested MDS resident data elements as covariates. We may lag some time-varying resident characteristics by one assessment because we want to study the associations of repositioning time with $\operatorname{PrU}$ development, controlling for the starting point of the resident for that assessment interval. Interaction variables will be included in the predictor data set.

Residents without PrUs who drop out of the study after at least a three-day stay but before the conclusion of 4 weeks will be included in the analysis based on intent to treat. Residents who develop a Stage 3 or worse PrU, or deep purple discoloration, will be removed from the study per repositioning protocol, but will be considered a completed case if the ulcer developed on the coccyx, sacrum, trochanter or heel. Residents who develop ulcers on sites not thought to be influenced by bed position or limited mobility, or related to sitting, will continue to be followed for analysis, but will not be included in the $\mathrm{NH}$-wide protocol.

Statistical power. According to previous research, the average $\mathrm{PrU}$ incidence rates range from $5.2-15 \%$. At the time of study design, the nine NHs within the NHS were identified with the highest PrU incidence rates which averaged 9.2\% ( $\mathrm{SD}=3.3 \%)$. In power analyses, we used $3.5 \%$ for PrU incidence as the expected rate for intervention since it is the highest rate found in the TURN study (for moderate-risk patients). The PrU incidence rates have been translated into an odds ratio of $0.38(=3.5 \%$ / 9.2\%) as the effect size to detect. For Aim 1, odds ratios from a logistic regression of $\operatorname{PrU}$ incidence rates in the three treatment arms (i.e., at 2-, 3-, and 4-h repositioning frequencies), controlling for risk level, are proposed to test the difference in PrU incidence rates between the 4-h arm and the 2-h arm as well as between the 4-h arm and the 3-h arm at 4 weeks; we are assuming that the distance between the 2-h arm and the TURN 3-h odds ratio will be larger. A sample size of $N$ $=951$ ( $n=317$ for each arm $)$ will be required to detect an odds ratio of .38 with a power of 0.95 at a one-tailed significance level of $\alpha=0.05$, according to G*Power. We are using a power of 0.95 instead of the conventional power of 0.80 to ensure that we will have a smaller Type II error to test the hypothesis of no-group difference that we expect to fail to reject. We are using a one-tailed test since we are only interested in determining whether the PrU rate is higher for assigned repositioning intervals longer than the current 2-h standard practice.. For Aim 2 , GEE is proposed. A sample size of $n=114$ for each group (total $N=342$ ) will be required to detect an effect size of $5.7 \%(=9.2 \%-3.5 \%)$ with a power of 0.80 at a one-tailed significance level of $\alpha=0.05$, using the algorithms GEESIZE developed by Dahmen, et al., on an autoregressive structure for residual correlations [48]. Since the nine NHs will be randomly assigned to the three treatment arms, risk level will be the only potential covariate to be controlled in the models, and this is already considered in the power analyses. Therefore, we will target a sample size of $N=951$ (the larger of the two required sample sizes) for both aims while taking into account potential losses due to turnover, attrition, and exclusion criteria. While this proposed study may look like a non-inferiority or equivalence trial, we will run regular one-tailed superiority tests, because (as noted earlier) we are only interested in examining whether or not the PrU rate is higher for less frequent repositioning than for the standard 2-h repositioning. (We assume that 2 -h repositioning was followed to produce the $9.2 \%$ 
average PrU incidence rate across our 9 study NHs, because that is the current standard of care). Also, according to Snapinn [49], non-inferiority trials have many controversial assumptions and limitations that need to be made or addressed, and that is beyond the control of our study. For incidence, it is difficult to specify a noninferiority margin for PrU rates. Instead, we will test the hypothesis of no group-difference by examining whether the 95\% confidence intervals of the odds ratios (for Aim 1 ) or the PrU rates (for Aim 2) of the two pairs of compared groups (e.g., 4-h arm vs. 2-h arm and 4-h arm vs. 3$\mathrm{h}$ arm) overlap. If the confidence intervals do overlap, the hypothesis for no-group difference will be confirmed.

Missing data. When data are missing, we will make adjustments depending on the variable and its intended use in the analyses. Residents with missing data will be deleted from analyses and continuous variables with missing data collapsed into categorical data, and cases with missing information for the continuous variable placed into a category using corroborating data. For example, we may not always have a resident's body mass index, but may have other weight- and height-related information (e.g., resident description as 'emaciated') that allows us to categorize a resident broadly, e.g., as underweight. We will use various types of imputation as appropriate and check assumptions about 'missing at random'. To exclude unrealistic values and obvious outliers from the analysis, we will set ranges for some variables, and values beyond those ranges will be deemed improbable and excluded. These values may also be sent back to the NHs for correction.

Qualitative analysis. NH staff and resident satisfaction with the intervention approach will be explored using semi-structured questions to staff and resident focus groups. A standardized topical guide will be used to evaluate 1) common perceptions related to satisfaction with the intervention approach, 2) areas of strength, and 3) areas for improvement. Verbatim transcripts will be checked against original recordings for accuracy. Data will be analyzed assisted by NVivo 11 along with field notes taken during the focus groups. As in the PI's prior clinical trial, transcripts will be analyzed using thematic content analysis; matrices used to organize the data and category summaries will be compared across $\mathrm{NHs}$ and staff and resident groups. A directed approach will identify core concepts, and the analytic plan will include use of satisfaction-based a priori codes and allow for new themes to emerge post hoc. Analytical rigor will assure that 1 ) the study measures what is intended (credibility), 2) results are similar when the work is repeated in the same way, in the same context with the same participants and methods (dependability), 3) the findings are the results of the experiences of the participants (confirmability), and 4) the results are applicable to other situations (transferability).

\section{Discussion}

This study will contribute to understanding of an optimal resident repositioning interval using VE surfaces for $\mathrm{NH}$ residents at different risk levels; further, the study will contribute to a much-needed understanding of the contribution of medical severity as a precipitating factor for PrU development. Effective prevention can potentially reduce PrU incidence and avoid treatment costs while improving overall resident safety and quality of life.

\section{Abbreviations}

ICER: Incremental cost-effectiveness ratio; ID: Identification; MDS: Minimum Data Set; NCAT: Nursing culture assessment tool; NH: Nursing home; NHS: Nursing home system; PM system: Patient management system; PrU: Pressure ulcer/injury; RMS: Risk Management System; VE: Visco-elastic

\section{Acknowledgements}

This project was funded by the National Institutes of Health, National Institute of Nursing Research (R01NR016001) Yap, PI.

Authors would like to thank Elizabeth Flint, PhD for her editing assistance.

\section{Funding}

Research reported in this publication is supported by the National Institute of Nursing Research of the National Institutes of Health under Award Number R01NR016001. The content is solely the responsibility of the authors and does not necessarily represent the official views of the National Institutes of Health.

\section{Availability of data and materials}

This paper does not include any data, as it is a protocol paper. When data is collected, we ask that readers please request it from the lead author.

\section{Authors' contributions}

TLY, SMK, SH wrote the protocol in consultation with NB, CC-E, SD who reviewed the protocols, executed them and assisted in refining the written protocols from the original proposal. All authors read and approved the final manuscript.

\section{Ethics approval and consent to participate}

The project was approved by Duke University IRB. Waiver of consent was granted because: 1) the entire cluster of low-, moderate-, and high-risk residents will receive a $\mathrm{NH}$-wide repositioning schedule, 2) the repositioning protocol will become part of $\mathrm{NH}$-wide practice standardizing the workflow of repositioning, 3) the cluster-level intervention involves minimal risk, and 4) extracted resident data from PM database, MDS, and EMR will be assigned a study identification (ID) number and coded data set will be placed directly into a secured network folder.

\section{Consent for publication}

Not applicable.

\section{Competing interests}

The authors declare that they have no competing interests.

\section{Publisher's Note}

Springer Nature remains neutral with regard to jurisdictional claims in published maps and institutional affiliations.

\section{Author details}

${ }^{1}$ School of Nursing, Duke University, Durham, NC, USA. ${ }^{2}$ College of Nursing, East Carolina University, Greenville, NC, USA. ${ }^{3}$ School of Medicine, University of Utah, Salt Lake City, Utah, USA. ${ }^{4}$ School of Nursing, University of Texas Health Science Center at Houston, Houston, TX, USA. ${ }^{5}$ Department of General Internal Medicine, Duke University, Durham, NC, USA. ${ }^{6}$ Division of Geriatrics, School of Medicine, Duke University, Durham, NC, USA. 
Received: 7 December 2017 Accepted: 7 February 2018 Published online: 20 February 2018

\section{References}

1. Centers for Medicare \& Medicaid Services (CMS). Action plan for (further improvement of) nursing home quality. Baltimore: Centers for Medicare \& Medicaid Services; 2008

2. Niederhauser A, VanDeusen LC, Parker V, Ayello EA, Zulkowski K, Berlowitz D. Comprehensive programs for preventing pressure ulcers: a review of the literature. Adv Skin Wound Care. 2012;25(4):167-88. quiz 189-190

3. Redelings MD, Lee NE, Sorvillo F. Pressure ulcers: more lethal than we thought? Adv Skin Wound Care. 2005;18(7):367-72.

4. Agency for Healthcare Research and Quality (AHRQ). Preventing pressure ulcers in hospitals: a toolkit for improving quality of care. Rockville: Agency for Healthcare Research and Quality; 2011.

5. Pham B, Stern A, Chen W, Sander B, John-Baptiste A, Thein HH, Gomes T, Wodchis WP, Bayoumi A, Machado M, et al. Preventing pressure ulcers in long-term care: a cost-effectiveness analysis. Arch Intern Med. 2011;171(20):1839-47.

6. Fife CE, Yankowsky KW, Ayello EA, Capitulo KL, Fowler E, Krasner DL, Mulder G, Sibbald RG. Legal issues in the care of pressure ulcer patients: key concepts for healthcare providers-a consensus paper from the international expert wound care advisory panel(c). Adv Skin Wound Care. 2010;23(11):493-507.

7. Lyder CH, Shannon R, Empleo-Frazier O, McGeHee D, White C. A comprehensive program to prevent pressure ulcers in long-term care: exploring costs and outcomes. Ostomy Wound Manage. 2002;48(4):52-62.

8. Keelaghan E, Margolis D, Zhan M, Baumgarten M. Prevalence of pressure ulcers on hospital admission among nursing home residents transferred to the hospital. Wound Repair Regen. 2008;16(3):331-6.

9. Reddy M, Gill SS. The effectiveness of pressure ulcer risk assessment instruments and associated intervention protocols remains uncertain. Evid Based Med. 2014;19(3):93.

10. Feng Z, Fennell ML, Tyler DA, Clark M, Mor V. The care span: growth of racial and ethnic minorities in US nursing homes driven by demographics and possible disparities in options. Health Aff (Millwood). 2011;30(7):1358-65.

11. Yap TL, Rapp MP, Kennerly S, Cron SG, Bergstrom N. Comparison study of Braden scale and time-to-erythema measures in long-term care. J Wound Ostomy Continence Nurs. 2015;42(5):461-7.

12. Bolton LL, Girolami S, Corbett L, van Rijswijk L. The Association for the Advancement of wound care (AAWC) venous and pressure ulcer guidelines. Ostomy Wound Manage. 2014;60(11):24-66.

13. National Pressure Ulcer Advisory Panel, European Pressure Ulcer Advisory Panel, Pan Pacific Pressure Injury Alliance. In: Haesler EE, editor. Prevention and treatment of pressure ulcers: clinical practice guideline. 2nd ed. Perth: Cambridge Media; 2014

14. Park-Lee E, Caffrey C. Pressure ulcers among nursing home residents: United States. NCHS data brief. 2004;2009(14):1-8.

15. Centers for Medicare \& Medicaid Services (CMS): MDS 3.0 Frequency Report. 2014

16. Bergstrom N. Litigation or redesign: improving pressure ulcer prevention. J Am Geriatr Soc. 2005:53(9):1627-9.

17. Braden B, Bergstrom N. A conceptual schema for the study of the etiology of pressure sores... Including commentary by Baggerly J and Phipps M... First printed in volume 12, number 1, January/February 1987. Rehabil Nurs. 2000;25(3):105-10.

18. Norton D, McLaren R, Exton-Smith AN. An investigation of geriatric nursing problems in hospital. Edinburgh: Churchill Livingston; 1962.

19. Defloor T, De Bacquer D, Grypdonck MH. The effect of various combinations of turning and pressure reducing devices on the incidence of pressure ulcers. Int J Nurs Stud. 2005:42(1):37-46.

20. Bergstrom N, Horn SD, Rapp MP, Stern A, Barrett R, Watkiss M. Turning for ulcer ReductioN: a multisite randomized clinical trial in nursing homes. J Am Geriatr Soc. 2013;61(10):1705-13.

21. Coleman S, Gorecki C, Nelson EA, Closs SJ, Defloor T, Halfens R, Farrin A, Brown J, Schoonhoven L, Nixon J. Patient risk factors for pressure ulcer development: systematic review. Int J Nurs Stud. 2013;50:974-1003.

22. Yap TL, Kennerly SM, Bergstrom N, Hudak SL, Horn SD. An evidence-based cue-selection guide and logic model to improve pressure ulcer prevention in long-term care. J Nurs Care Qual. 2016;31(1):75-83.
23. Horn SD, Gassaway J. Practice-based evidence study design for comparative effectiveness research. Med Care. 2007:45(10 Supl 2):S50-7.

24. Horn SD, Bender SA, Ferguson ML, Smout RJ, Bergstrom N, Taler G, Cook AS, Sharkey SS, Voss AC. The National Pressure Ulcer Long-Term Care Study: pressure ulcer development in long-term care residents. J Am Geriatr Soc. 2004;52(3):359-67.

25. Braden BJ, Bergstrom N. Clinical utility of the Braden scale for predicting pressure sore risk. Decubitus. 1989;2(3):44-6. 50-41

26. Pieper B. Long term care/nursing home. In: Pieper B, National Pressure Ulcer Advisory Panel (NPUAP), editors. Pressure ulcers: prevalence, incidence and implications for the future. 2nd ed. Washington: National Pressure Ulcer Advisory Panel (NPUAP); 2012. p. 65-96.

27. Cuddigan J, Berlowitz DR, Ayello EA, National Pressure Ulcer Advisory Panel Board of Directors. Pressure ulcers in America: prevalence, incidence, and implications for the future. An executive summary of the National Pressure Ulcer Advisory Panel monograph. Adv Skin Wound Care. 2001;14(4, Part 1 of 2):208-15.

28. Abel RL, Warren K, Bean G, Gabbard B, Lyder CH, Bing M, McCauley C. Quality improvement in nursing homes in Texas: results from a pressure ulcer prevention project. J Am Med Dir Assoc. 2005;6(3):181-8.

29. Zulkowski K, Langemo D, Posthauer ME. Coming to consensus on deep tissue injury. Adv Skin Wound Care. 2005;18(1):28-9.

30. Rosen J, Mittal V, Degenholtz H, Castle N, Mulsant BH, Nace D, Rubin FH. Pressure ulcer prevention in black and white nursing home residents: a Q initiative of enhanced ability, incentives, and management feedback. Adv Skin Wound Care. 2006;19(5):262-8.

31. Yap TL, Kennerly SM, Simmons MR, Buncher CR, Miller E, Kim J, Yap WY. Multidimensional team-based intervention using musical cues to reduce odds of facility-acquired pressure ulcers in long-term care: a paired randomized intervention study. J Am Geriatr Soc. 2013:61(9):1552-9.

32. Bergstrom N, Braden BJ, Laguzza A, Holman V. The Braden scale for predicting pressure sore risk. Nurs Res. 1987;36(4):205-10.

33. Moore ZE, Cowman S. Repositioning for treating pressure ulcers. Cochrane Database Syst Rev. 2009;2:CD006898.

34. Bates-Jensen BM, Alessi CA, Cadogan M, Levy-Storms L, Jorge J, Yoshii J, Al-Samarrai NR, Schnelle JF. The minimum data set bedfast quality indicator: differences among nursing homes. Nurs Res. 2004;53(4):260-72.

35. Bates-Jensen BM, Simmons SF, Schnelle JF, Alessi C. Evaluating the accuracy of minimum data set bed-mobility ratings against independent performance assessments: systematic error and directions for improvement. Gerontologist. 2005;45(6):731-8.

36. Saliba D, Rubenstein LV, Simon B, Hickey E, Ferrell B, Czarnowski E, Berlowitz $D$. Adherence to pressure ulcer prevention guidelines: implications for nursing home quality. J Am Geriatr Soc. 2003;51(1):56-62.

37. Documentation tops provider concerns in survey [https://www. iadvanceseniorcare.com/news-item/documentation-tops-provider-concernssurvey].

38. National Healthcare Quality and Disparities Reports [http://www.ahrq.gov/ research/findings/nhardr/nhardr11/10 functional_status/T10 1 6 6 4.html].

39. National Pressure Ulcer Advisory Panel (NPUAP), European Pressure Ulcer Advisory Panel (EPUAP). International pressure ulcer guidelines for prevention and treatment: clinical practice guidelines. Washington: National Pressure Ulcer Advisory Panel (NPUAP); 2009.

40. Yap TL, Kennerly SM, Corazzini K, Porter C, Toles M, Anderson RA. Evaluation of cueing innovation for pressure ulcer prevention using staff focus groups. Healthcare. 2014;2(3):299-314.

41. Medicare.gov Nursing Home Compare [http://www.medicare.gov/ NursingHomeCompare/search.aspx?bhcp=1].

42. Bergstrom N, Braden B. A prospective study of pressure sore risk among institutionalized elderly. J Am Geriatr Soc. 1992;40(8):747-58.

43. Bellg AJ, Borrelli B, Resnick B, Hecht J, Minicucci DS, Ory M, Ogedegbe G, Orwig D, Ernst D, Czajkowski S, et al. Enhancing treatment fidelity in health behavior change studies: best practices and recommendations from the NIH behavior change consortium. Health Psychol. 2004;23(5):443-51.

44. Larson B. A pilot study to assess the DynaSense system. CA: Leaf Healthcare, Inc. Clinical Trials.gov Identifier NCT02005692; 2013.

45. Larson B. Leaf patient monitor validation human clinical study report. Internal document PN 1547AA. CA: Leaf Healthcare, Inc; 2014.

46. Yap TL, Kennerly S, Flint EP. Nursing Culture Assessment Tool (NCAT): Empirical validation for use in long-term care International Journal of Nursing Sciences. 2014;1(3):241-249. 
47. Kennerly S, Heggestad E, Myers H, Yap TL. Using the Nursing Culture Assessment Tool (NCAT) in long-term care: An update on psychometrics and scoring standardization. Healthcare. 2015;3:637-647. Open access: http:// www.mdpi.com/2227-9032/3/3/637.

48. Dahmen G, Rochon J, Konig IR, Ziegler A. Sample size calculations for controlled clinical trials using generalized estimating equations (GEE). Methods Inf Med. 2004;43(5):451-6.

49. Snapinn SM. Noninferiority trials. Curr Control Trials Cardiovasc Med. 2000;1(1):19-21.

Submit your next manuscript to BioMed Central and we will help you at every step:

- We accept pre-submission inquiries

- Our selector tool helps you to find the most relevant journal

- We provide round the clock customer support

- Convenient online submission

- Thorough peer review

- Inclusion in PubMed and all major indexing services

- Maximum visibility for your research

Submit your manuscript at www.biomedcentral.com/submit 